ANNE BOHNENKAMP

\title{
Goethes poetische Orientreise
}

\author{
Vorblatt
}

\section{Publikation}

Erstpublikation in: Goethe-Jahrbuch 120/2003, S. 144-156.

Neupublikation im Goethezeitportal

Vorlage: Datei der Autorin

URL:

$<$ http://www.goethezeitportal.de/db/wiss/goethe/divan_bohnenkamp.pdf $>$ Eingestellt am 18.10.2004

\section{Autorin}

PD Dr. Anne Bohnenkamp-Renken

Freies Deutsches Hochstift

Großer Hirschgraben 23-25

60311 Frankfurt a.M.

Emailadresse: <abohnenkamp@goethehaus-frankfurt.de>

\section{Empfohlene Zitierweise}

Beim Zitieren empfehlen wir hinter den Titel das Datum der Einstellung oder des letzten Updates und nach der URL-Angabe das Datum Ihres letzten Besuchs dieser Online-Adresse anzugeben:

Anne Bohnenkamp-Renken: Goethes poetische Orientreise (18.10.2004). In: Goethezeitportal. URL:

$<$ http://www.goethezeitportal.de/db/wiss/goethe/divan_bohnenkamp.pdf $>$ (Datum Ihres letzten Besuches). 


\title{
ANNE BOHNENKAMP
}

\section{Goethes poetische Orientreise}

\author{
„Aber ist denn Bagdad \\ so weit?"“1
}

$\mathrm{Da} 3$ man auf sehr unterschiedliche Weise und zu ganz unterschiedlichen Zwecken reisen kann, dafür bietet Goethes Leben und Werk reiches Anschauungsmaterial. Wie es im Deutschen Wörterbuch heißt, ist „die gewöhnliche bedeutung von reise in der ältern sprache, Aufbruch zum kriege, der kriegszug selbst ${ }^{\text {‘2 }}$ und auch diese - wortgeschichtlich gesehen - 'Urform' des Reisens finden wir auch bei Goethe: bekanntlich begleitete er 1792 seinen Herzog auf dem Feldzug nach Frankreich und mußte für diesen Zweck Christiane und den noch nicht dreijährigen August alleine zuhause lassen, was ihm wenig gefiel. Aber auch Dienstreisen anderer Art unternahm der Geheimrat im Auftrag seines Herzogs. ${ }^{3}$ Eine wichtige Rolle spielen seit 1785 bekanntlich auch Goethes Kur- und Badereisen, die der Erholung und Regeneration gewidmet waren. Berühmt sind vor allem aber seine Studien- oder Bildungsreisen, zu denen die Reisen in die Schweiz und natürlich die Reise nach Italien zu rechnen sind. Als Mittel der für eine Reise konstitutiven Fortbewegung hat Goethe verschiedene Vehikel eingesetzt, das Spektrum reicht von den Fußwanderungen über die Fortbewegung zu Pferd, zu Schiff oder in der Kutsche bis zu den Reisen, die Goethe im Geiste unternahm - ausschließlich mit den Flügeln der eigenen Vorstellungskraft oder auch mit der Hilfe von Büchern, inspiriert durch Lektüre.

$\mathrm{Zu}$ dieser letzten Art Reisen gehört offensichtlich die, um die es im folgenden gehen soll: „Goethes poetische Orientreise“. Der Titel bezeichnet Goethes erstaunliche Begegnung mit den Dichtern des Orients in den Jahren von 1814 bis 1819 und das Werk, das daraus entstand. Es geht also um den Westöstlichen Divan, der mindestens in doppelter Hinsicht eine 'poetische' Reise ist: eine Reise hin zu der Poesie des Orients und eine Reise mit den Mitteln der Poesie: der Phantasie und der literarischen Gestaltung.

\footnotetext{
${ }^{1}$ Diese Frage aus einem Nachlassgedicht (Hudhud, als einladender Bote. FA I, 3.1, S. 613) spielt an auf einen Vers aus dem Buch Suleika, der die Antwort zu geben scheint: „Für Liebende ist Bagdad nicht weit" (FA I, 3, S. 88). Während der Arbeit an diesem Vortrag wurde Bagdad von amerikanischen Streitkräften bombardiert.

${ }^{2}$ Deutsches Wörterbuch von Jacob Wilhelm Grimm. Bearb. von und unter Leitung von Dr. Moritz Heyne. München 1984 [Fotomechan. Nachdruck d. Erstausgabe 1893-1924), Bd. 14 [= Bd. 8], S. 718.

${ }^{3}$ So etwa verschiedene Reisen im Zusammenhang mit Fragen des Bergbaus oder auch die zweite Reise nach Italien, als er 1790 die Herzoginmutter in Venedig traf und nach Hause geleitete.
} 
Beide Teile des West-östlichen Divans, die Versammlung der Gedichte selbst und der diese in größere Zusammenhänge stellende Prosateil, beginnen mit dem Aufbruch zu einer Reise: „Flüchte Du, im reinen Osten | Patriarchenluft zu kosten“" (FA I, 3.1, S. 12) heißt es im eröffnenden Gedicht Hegire, das den Band mit diesem Titel unter das Zeichen einer spezifisch motivierten Reise stellt: unter das Zeichen einer Flucht, die gleichzeitig den Beginn einer neuen Zeitrechnung markiert. Neben der dominanten Bezugnahme auf Mohammeds Emigration von Mekka nach Medina im Jahr 622 kommt hier auch Goethes Italienreise ins Spiel, die er ebenfalls als „Hegire“ bezeichnet hat. ${ }^{4}$ Wie damals, als Goethe 1786 heimlich aus Karlsbad aufbrach, treibt ihn auch 1814 eine als unbefriedigend empfundene Gegenwart aus dem Lande: „Ich segne meinen Entschluß zu dieser Hegire, denn ich bin dadurch der Zeit und dem lieben Mittel-Europa entrückt, welches für eine große Gunst des Himmels anzusehen ist, die nicht einem jeden widerfährt" (an Knebel am 8.2.1815, WA IV, 25, S. 190).

Unter dem Motto „Wer das Dichten will verstehen | Muß in’s Land der Dichtung gehen; | Wer den Dichter will verstehen | Muß in Dichters Lande gehen“" (West-östlicher Divan, FA I, 3.1, S. 137) setzt dann auch der Besserem Verständniss gewidmete zweite Teil ein mit der Aufforderung zum Aufbruch in andere Länder, die nun jedoch anders begründet wird: die vorangestellten Verse erklären das Reisen zur notwendigen Bedingung des Verstehens. Die folgende Einleitung entwickelt eine entsprechende Fiktion vom Autor als Reisendem, die ausdrücklich als solche reflektiert wird ${ }^{5}$ : „Am liebsten aber wünschte der Verfasser vorstehender Gedichte als ein Reisender angesehen zu werden, dem es zum Lobe gereicht, wenn er sich der fremden Landesart mit Neigung bequemt, deren Sprachgebrauch sich anzueignen trachtet, Gesinnungen zu theilen, Sitten aufzunehmen versteht“". (FA I, 3.1, S. 138)

Anders als sein empirischer Autor, der gleichzeitig ja in die entgegengesetzte Richtung zu einer Reise an Rhein und Main nach Westen aufbricht ${ }^{6}$,

4 „meine Hegire aus Karlsbad“-Italienische Reise (FA I, 15, S. 429f.). Vgl. auch an Carl August, 14.10.1786 (WA IV, 8, S. 33).

${ }^{5}$ Es handelt sich im Divan also nicht um einen jener Reiseberichte, für die die Frage nach der Authentizität im Vagen bleibt, wie das in zeitgenössischen Reiseberichten durchaus üblich war. Vgl. Mirjam Weber: Der ,, wahre Poesie-Orient“. Eine Untersuchung zur Orientalismus-Theorie Edward Saids am Beispiel von Goethes ,, West-östlichen Divan “ und der Lyrik Heines. Wiesbaden 2001, S. 14: „Bei der Produktion wie der Rezeption von Reiseliteratur haben häufig weder Leser noch Autoren zwischen realen und imaginären Reisen unterschieden: Fiktive, konstruierte Berichte traten mit dem gleichen Anspruch auf Authentizität auf wie Schilderungen empirisch erfahrbarer Realität." Goethes Divan ist dagegen ausdrücklich eine Reise im Modus des 'als ob', eine vorgestellte, eine dichterische Reise.

${ }^{6} \mathrm{Da}$ die imaginäre Reise im Entstehungsprozeß des Divan mit Goethes ganz realen Reisen an Rhein und Main zusammenfällt - die beiden handlichen Hafis-Bändchen, die Goethe gerade von seinem Verleger Cotta geschenkt bekommen hatte, steckte er als Reiselektüre ein, als er am 25. Juli 1814 mit der Kutsche von Weimar nach Westen aufbrach - hat vielfache Spuren hinterlassen: der 'hybride' Effekt einer stereoskopen Wahrnehmung beider Reisen in einer ist am deutlichsten dem Gedicht Liebliches (FA I, 3, S. 19f.) eingeschrieben, das unter Anspielung auf Goethes viele Jahre zurückliegende Übersetzung eines serbischen Liedes die imagi- 
ist der „Verfasser vorstehender Gedichte“ als fiktiver Orientreisender nicht in der Kutsche unterwegs, sondern zu Fuß - „Jeden Pfad will ich betreten | Von der Wüste zu den Städten“ (Hegire. In: FA I, 3.1, S. 12) - oder auf dem Rücken eines Reittiers: „Und ich reite froh in alle Ferne, | Ueber meiner Mütze nur die Sterne“ (Freysinn. In: FA I, 3.1, S. 14). Der Dichter inszeniert sich dabei als ein Handelsreisender: „Damit aber alles was der Reisende zurückbringt den Seinigen schneller behage, übernimmt er die Rolle eines Handelsmanns, der seine Waaren gefällig auslegt und sie auf mancherley Weise angenehm zu machen sucht; ankündigende, beschreibende, ja lobpreisende Redensarten wird man ihm nicht verargen“ (FA I, 3.1, S. 139) heißt es in einer charakteristischen metapoetischen Reflexion der schon zitierten Einleitung zum Prosateil. Diese „Rolle eines Handelsmanns“ übernimmt der Dichter nicht nur in dem schon zitierten Eröffnungsgedicht Hegire ${ }^{7}$, sondern besonders anschaulich und detailreich auch im Buch Suleika, in dessen zwischen Main und Euphrat changierendem „Duo-Drama“ ${ }^{\text {8 }}$ der Liebende als reisender Händler auftritt: „Mich, der von den Indostanen | Streifte bis Damascus hin, Um mit neuen Caravanen | Bis an's rothe Meer zu ziehn. ${ }^{9} \mid$ Mich vermählst du deinem Flusse, | Der Terrasse, diesem Hayn, | Hier soll bis zum letzten Kusse | Dir mein Geist gewidmet seyn.“ (West-östlicher Divan. FA I, 3.1, S. 77). In der hyperbolischen Überbietungsmetaphorik des Gedichtes Nur wenig ist's was ich verlange legt der Dichter die Gewinne des ,ganzen Handelswesens“ dann der Geliebten zu Füßen. Von den Kostbarkeiten der orientalischen Welt - Edelsteine, Früchte, Stoffe, Gewürze bringt ,alles was die Welt ergetzte | Die Caravane dir heran“" (FA I, 3.1, S. 81f.). Wie im Prosateil wird jedoch auch im Gedicht deutlich, daß das alles nicht in der Wirklichkeit geschieht, sondern in der Phantasie des liebenden Dichters: „Oft sitz' ich heiter in der Schenke | Und heiter im beschränkten Haus; | Allein so bald ich dein gedenke; | Dehnt sich mein Geist erobernd aus“ (Ebd.). Die Schätze, die der handlungsreisende Dichter unterwegs erwirbt, sind poetische Schätze; die Perlen, die Hatem Suleika verehrt, „dichtrische Perlen“ (Die schön geschriebenen. In: FA I, 3.1, S. 83). Die Handelsware, um die es hier geht, sind also Gedichte; das Handelswesen gilt der Poesie, dem literarischen Austausch.

nierte östliche und die reale westliche Landschaft ineinander blendet. Daß dieser Westen ganz konkret Goethes Thüringen ist, verrät die erhaltene Erstfassung des Beginns der vierten Strophe: „Ja es sind die bunten Mohne, | Die um Erfurt sich erstrecken“ (FA I, 3, S. 476 und S. 940).

7 „Wenn mit Caravanen wandle, | Schawl, Caffee und Moschus handle.“ FA I, 3, S. 12.

${ }^{8}$ So Goethes Charakterisierung dieses Buches in seiner Ankündigung des Divans im Morgenblatt für gebildete Stände. In: FA I, 3, S. 550.

${ }^{9}$ Die Verse bezeichnen das ,traditionelle - von den Reichen Vorderindiens über die omajjadische Hauptstadt Damaskus bis nach Mekka und nach Ägypten reichende arabische Handelsgebiet" (Hendrik Birus in: FA I, 3.2, S. 1188). 
Das für den West-östlichen Divan zentrale Bildfeld der poetischen Handelsreise stellt Goethes letzte große Gedichtsammlung sehr deutlich in einen Zusammenhang mit seiner Idee einer 'Weltliteratur', wie er sie ein gutes Jahrzehnt nach den Divan-Jahren entwickelte - zu einer Zeit, als im Rahmen der ,Ausgabe letzter Hand' gerade die zweite erweiterte Ausgabe des West-östlichen Divans erschien. 'Weltliteratur' im Sinne Goethes bezeichnet in Analogie zum internationalen Warenaustausch der Kaufleute den „Wechseltausch“10 von Kulturgütern zwischen den Völkern; abweichend vom heute dominierenden Wortgebrauch meint Goethe mit 'Weltliteratur' ja nicht die Summe aller literarischen Erzeugnisse oder einen Kanon weltweit und überzeitlich gültiger literarischer Werke, sondern eine Tätigkeit der Kommunikation und des Austauschs ${ }^{11}$, den er wiederholt als ,mehr oder weniger freyen geistigen Handelsverkehr“"12 oder auch als „Freihandel der Begriffe und Gefühle“ beschrieben hat. ${ }^{13}$ Auf dem Weg zu diesem Konzept des alten Goethe stellt der Divan einen entscheidenden Schritt dar; er bietet ein frühes Beispiel für solchen interkulturellen und internationalen literarischen Austausch, wie Goethe ihn später als 'Weltliteratur' konzipiert hat. ${ }^{14} \mathrm{Ja}$, ohne Goethes Orienterfahrung hätte es das Konzept der 'Weltliteratur' so nicht gegeben.

Die engen Verbindungen zwischen dem West-östlichen Divan und der Idee einer 'Weltliteratur' werden auch in der zentralen Rolle ganz deutlich, die für beide das Übersetzen spielt. Für den Austausch zwischen den „Litteratoren und Litteraturen“"15 verschiedenster Weltgegenden über Nationalitäts- und Sprachgrenzen hinweg, für Goethes 'Weltliteratur' also, kann das Übersetzen geradezu als paradigmatische Tätigkeit gelten: der Übersetzer ist der eigentli-

\footnotetext{
${ }^{10}$ German Romance. In: Kunst und Alterthum VI 2. FA I, 22, S. 434.

${ }^{11}$ Das von Goethe in Umlauf gebrachte Kompositum stellte er 1827 und 1828 in den Heften seiner Zeitschrift Über Kunst und Alterthum vor (Vgl. FA I, 22, S. 293-520 sowie Bohnenkamp, 'Den Wechseltausch zu befördern'. Goethes Entwurf einer Weltliteratur. In: FA I, 22, S. 937-964).

${ }^{12}$ So im Vorwort zu Carlyles „Leben Schillers“. In: Aufsätze, Entwürfe Notizen 1824-1832. FA I, 22, S. 870: „Es ist schon einige Zeit von einer allgemeinen Weltliteratur die Rede und zwar nicht mit Unrecht: denn die sämmtlichen Nationen, in den fürchterlichsten Kriegen durcheinander geschüttelt, sodann wieder auf sich selbst einzeln zurückgeführt, hatten zu bemerken, daß sie manches Fremde gewahr worden, in sich aufgenommen, bisher unbekannte geistige Bedürfnisse hie und da empfunden. Daraus entstand das Gefühl nachbarlicher Verhältnisse, und anstatt daß man sich bisher zugeschlossen hatte, kam der Geist nach und nach zu dem Verlangen, auch in den mehr oder weniger freyen geistigen Handelsverkehr mit aufgenommen zu werden.“

${ }^{13}$ Nach dem Bericht von A.E. Odyniec. In: Flodoard von Biedermann: Goethes Gespräche. Eine Sammlung zeitgenössischer Berichte aus seinem Umgang. Auf Grund der Ausg. und des Nachlasses von Flodoard von Biedermann erg. und hrsg. von Wolfgang Herwig. Zürich / Stuttgart / München 1972, Bd. 3.2, S. 471.

${ }^{14}$ Dieser Zusammenhang ist in der Forschung auch immer wieder gesehen worden. Vgl. neuerdings ausführlich die Studie von Manfred Koch, Weimaraner Weltbewohner. Zur Genese von Goethes Begriff, Weltliteratur'. Tübingen 2002.

${ }^{15}$ Die hier zitierte Tagebuchnotiz vom 8.5.1826 steht im Kontext der Entwicklung der Idee einer Weltliteratur: „Einiges dictirt über mein Verhältniß zu fremden Litteratoren und Litteraturen“ (WA III,10,189).
} 
che Handelsreisende in Sachen Literatur. Besonders deutlich wird das in einem Aufsatz über Thomas Carlyles Übersetzungen deutscher Texte, in dem Goethe die große Bedeutung von Übersetzungen für den ,allgemeinen Weltverkehr“ herausstellt und den Übersetzer als „,Vermittler dieses allgemein geistigen Handels“" charakterisiert, der ,auf dem Markte wo alle Nationen ihre Waaren anbieten [...] den Wechseltausch zu befördern sich zum Geschäft macht“. ${ }^{16}$ Im Westöstlichen Divan sind Übersetzungen bekanntlich ebenfalls von großer Bedeutung. Sie spielen nicht nur für die konkrete Entstehung der Divan-Gedichte eine entscheidende, dabei im Einzelnen ganz unterschiedliche Rolle, sondern die Übersetzer und das Übersetzen nehmen auch einen wichtigen Platz ein im Prosateil des West-östlichen Divans. In der zweiten Hälfte der Noten und Abhandlungen, die mit einer Folge von Kapiteln zu Reisenden und Lehrern den Goethe vorangegangenen Vermittlern zwischen Orient und Okzident gewidmet ist, kulminiert die Darstellung keineswegs zufällig in einem Kapitel, das unter dem Titel Übersetzungen Goethes wichtigste übersetzungstheoretische Überlegung enthält und das den verschiedenen möglichen Verfahren des Übersetzens gewidmet ist. (FA I, 3.1, S. 268-283)

Abweichend von der in diesem Zusammenhang üblichen Dichotomie, wie sie Goethe 1813 selbst in seiner Rede zum Andenken Wielands vorstellt und wie sie auch aus Schleiermachers Akademie-Rede aus dem gleichen Jahr, Ueber die verschiedenen Methoden des Uebersetzens, bekannt ist $^{17}$, unterscheidet

${ }^{16}$ German Romance. In: Kunst und Alterthum VI 2. FA I 22, S. 434. Daß es bei diesem 'Wechseltausch' um 'Weltliteratur' geht, kommt ausdrücklich in einem Schreiben an Carlyle vom 1. Januar 1828 zum Ausdruck, den Goethe um die Beurteilung einer Übertragung seines Tasso in das Englische bittet: „Nun aber möcht ich von Ihnen wissen, inwiefern dieser Tasso als Englisch gelten kann. Sie werden mich höchlich verbinden, wenn Sie mich hierüber aufklären und erleuchten; denn eben diese Bezüge vom Originale zur Übersetzung sind es ja, welche die Verhältnisse von Nation zu Nation am allerdeutlichsten aussprechen und die man zu Förderung der vor- und obwaltenden allgemeinen Weltliteratur vorzüglich zu kennen und zu beurtheilen hat.“ (WA IV, 43, S. 222) Vgl. dazu auch Goethe an Boisserée am 24. 4. 1831 (WA IV, 48, S. 188-191). - Den Gewinn, den Goethe sich von dem durch Übersetzungen geförderten „Wechseltausch“ verspricht, erläutert er mit Bezug auf das ebenfalls in Kunst und Alterthum VI 2 besprochene Leben Schillers von Carlyle im Gespräch mit Eckermann am 15. 7. 1827: „Es ist aber sehr artig, daß wir jetzt, bei dem engen Verkehr zwischen Franzosen, Engländern und Deutschen, in den Fall kommen, uns einander zu korrigieren. Das ist der große Nutzen, der bei einer Weltliteratur herauskommt und der sich immer mehr zeigen wird. Carlyle hat das Leben von Schiller geschrieben und ihn überhaupt so beurteilt, wie ihn nicht leicht ein Deutscher beurteilen wird. Dagegen sind wir über Shakespeare und Byron im klaren und wissen deren Verdienste vielleicht besser zu schätzen als die Engländer selbst." (FA II, 12, S. 257)

${ }^{17}$ Schleiermacher stellt in seiner berühmten Rede aus dem Sommer 1813 zwei Wege vor, die der Übersetzer einschlagen kann, um ,seinen Schriftsteller und seinen Leser [...] einander zuzuführen“: „,Entweder der Uebersetzer läßt den Schriftsteller möglichst in Ruhe, und bewegt den Leser ihm entgegen; oder er läßt den Leser möglichst in Ruhe, und bewegt den Schriftsteller ihm entgegen." Friedrich Daniel Ernst Schleiermacher: Ueber die verschiedenen Methoden des Uebersetzens. In: ders.: Akademievorträge. Hg. von Martin Rössler. (Bd. 11 der ersten Abteilung (Schriften und Entwürfe) der Kritischen Gesamtausgabe Schleiermacher), Berlin / New York 2002, S. 67-93. Goethe formuliert diese traditionsreiche überset- 
Goethe im West-östlichen Divan drei mögliche Vorgehensweisen. Während die ersten beiden „Arten der Uebersetzung“ , „eine schlicht-prosaische“ (die „uns in unserem eigenen Sinne mit dem Auslande bekannt macht“) und die ,parodistische“ (wo man ,nur fremden Sinn sich anzueignen und mit eignem Sinne wieder darzustellen bemüht ist", FA I, 3.1, S. 280, - Goethe nennt Luther und Wieland als Beispiele), keine größeren Verständnisschwierigkeiten machen, ist die Deutung der dritten Art ,wo man die Uebersetzung dem Original identisch machen möchte" (FA I, 3.1, S. 281), von Goethe dem 'höchsten und letzten' Zeitraum zugeordnet, bis heute umstritten. ${ }^{18}$ Es spricht jedoch vieles dafür nicht zuletzt, daß Goethe für dieses dritte Verfahren die Übertragungen von Johann Heinrich Voß als Beispiel anführt -, daß der Übersetzungstyp, den er hier vor Augen hatte, mit dem von Schleiermacher beschriebenen Verfahren 'den Leser zum Schriftsteller zu bringen’ konvergiert. „Diese Art“, so Goethe, ,erlitt anfangs den größten Widerstand; denn der Uebersetzer der sich fest an sein Original anschließt giebt mehr oder weniger die Originalität seiner Nation auf, und so entsteht ein Drittes, wozu der Geschmack der Menge sich erst heran bilden muß“ (West-östlicher Divan. FA I, 3.1, S. 281).

Im Gegensatz zu den ersten beiden Verfahren, die sich als Spielarten der Methode beschreiben lassen, 'den Schriftsteller zum Leser zu bringen', indem sie, auf unterschiedliche Weise, etwas Eigenes an die Stelle des Fremden setzen und das Fremde damit so 'einbürgern', daß es als Fremdes möglichst nicht mehr zu erkennen ist, ${ }^{19}$ lässt sich das dritte Verfahren dadurch kennzeichnen, daß es die grundsätzlich allem Übersetzen eigene Hybridität als solche sichtbar hält, statt sie zu verschleiern. ${ }^{20}$ Solches Übersetzen verhält sich damit umgekehrt analog zu dem Reisenden, den wir als den „Verfasser vorstehender Gedichte“ kennengelernt haben und der sich zwar „der fremden Landesart mit Neigung bequemt“ und ihren „Sprachgebrauch“, ihre „Gesinnungen“ und „Sitten“ aufzunehmen trachtet, aber doch, so heißt es in der Einleitung weiter, ,immer noch an einem eignen Accent, an einer unbezwingli-

zungstheoretische Alternative in seiner Wieland-Rede aus dem Februar des gleichen Jahres so: "Es giebt zwey Uebersetzungsmaximen. Die eine verlangt, daß der Autor einer fremden Nation zu uns herüber gebracht werde, dergestalt, daß wir ihn als den unsrigen ansehen können; die andere dagegen macht an uns die Forderung, daß wir uns zu dem Fremden hinüber begeben und uns in seine Zustände, seinen Styl, seine Eigenheiten finden sollen." ("Rede zu Wielands Andenken", 18. 2. 1813, FA I 17, S. 438).

${ }^{18} \mathrm{Vgl}$. Antonella Nicoletti: Übersetzung als Auslegung in Goethes ,, West-östlichem Divan “ im Kontext frühromantischer Übersetzungstheorien und Hermeneutik. Tübingen und Basel 2002, Diss. Uni. Basel, (Basler Studien zur deutschen Sprache und Literatur; Bd. 18), bes. S. 105-137.

${ }^{19}$ Als weitgehend unangefochtenes Ideal der literarischen Übersetzung gilt bis heute, so zu übersetzen, daß sich der übersetzte Text liest 'wie ein Original'. Vgl. u.a. Jiří Levý: Die literarische Übersetzung. Theorie einer Kunstgattung. Ins Deutsche übertragen von Walter Schamschula. Frankfurt a. M. / Bonn 1969.

${ }^{20}$ Zur 'hybriden' Übersetzung vgl. Anne Bohnenkamp: „,Hybrid“ statt ,, verfremdend“. Überlegungen zu einem Topos der Übersetzungstheorie. In: Linguistik in der Übersetzungswissenschaft. Hrsg. von Peter Colliander, Doris Hansen und Ingeborg Zint-Dyhr. Tübingen 2003, S. 9-26. 
chen Unbiegsamkeit seiner Landsmannschaft als Fremdling kenntlich bleibt.“ (West-östlicher Divan. FA I, 3.1, S. 138f.). Solche Übersetzung holt das fremde Werk zwar in die eigene Sprache hinüber, aber so, daß sie, um noch einmal Schleiermacher zu zitieren, ,ahnden läßt, daß sie nicht ganz frei gewachsen, vielmehr zu einer fremden Aehnlichkeit hinübergebogen sey ${ }^{\text {‘21 }}$. Deutlich wird, daß die verschiedenen Übersetzungsverfahren grundsätzlich unterschiedliche Haltungen gegenüber dem Fremden implizieren, ja, mit Klaus Reichert läßt sich sagen: hinter den ,entgegengesetzten Forderungen ans Übersetzen verbirgt sich [...] eine Kulturtheorie, eine heimliche Theorie über den Umgang mit dem Fremden".22

$\mathrm{Zu}$ fragen wäre nun, wie sich Goethes Vorstellung von 'Weltliteratur' zu diesen Übersetzungen der dritten Art verhält und welche Art des Übersetzens für Goethes eigenen Umgang mit dem Fremden im West-östlichen Divan charakteristisch ist. Während Bernd Witte in einem Aufsatz von 2001 konstatiert: „Es ist evident, daß Goethe mit diesen Übersetzungen der dritten Art auch auf seine eigenen Bemühungen um den Divan des persischen Dichters Hafis zielt ${ }^{\text {‘23, }}$, kommen andere Untersuchungen zum West-östlichen Divan zu dem Ergebnis, daß für Goethe die Forderung ,das Fremde in seinem Fremdsein zu belassen“ „historisch außerhalb seiner Möglichkeiten lag“24 und daß Edward Saids Beschreibung der westlichen Beschäftigung mit dem Orient als „Orientalism“ einer Sichtweise auf den Orient, die eigene Vorurteile projiziert, statt das Andere als solches wahrzunehmen - mit gewissen Einschränkungen auch Goethes West-östlichen Divan trifft, dessen Umgang mit dem Orient als Anverwandlung und Einbürgerung beschrieben wird. ${ }^{25}$

\footnotetext{
${ }^{21}$ Schleiermacher (Anm. 17) S. 81.

${ }^{22}$ Klaus Reichert: Zur Übersetzbarkeit von Kulturen - Appropriation, Assimilation oder ein Drittes? In: ,Zwischen den Kulturen. “ Theorie und Praxis des interkulturellen Dialogs. Hrsg. von Carola Hilfrich-Kunjappu und Stephane Mosès. Tübingen 1997, S. 35.

${ }^{23}$ Bernd Witte: ,,Hegire“. Transkulturelle Übersetzung in Goethes , West-oestlichem Divan'. In: Dagmar Ottmann, Markus Symmank (Hrsg.), Poesie als Auftrag. Festschrift für Alexander von Bormann. Würzburg 2001, S. 83-92, hier S. 84.

${ }^{24}$ Vgl. Stefan Blessin („Goethes West-östlicher Divan und die Entstehung der Weltliteratur“. In: West-östlicher und nordsüdlicher Divan. Goethe in interkultureller Perspektive. Hrsg. von. Ortrud Gutjahr. Paderborn / München [u.a.] 2000, S. 59-71), der bei Goethe ein Verfahren der 'produktiven Anverwandlung' erkennt, das im Rahmen heute geforderter „Interkulturalität" nicht mehr am Platze sei: „Goethe in interkultureller Perspektive gibt es strenggenommen nicht.“ (Ebd., S. S. 69 u. 70).

${ }^{25}$ So bei Mirjam Weber (Anm. 5), S. 13-28. Vgl. aber Hendrik Birus (Goethes imaginativer Orientalismus. In: Jahrbuch des Freien Deutschen Hochstifts 1992, S. 107-128), der ausgehend von Saids Untersuchung ein differenzierteres Bild von Goethes ,,spielerischreflektiertem Umgang mit seiner eigenen, zwischen Exteriorität und Empathie oszillierenden, west-östlichen Position gegenüber der Welt und Dichtung des Orients“ zeichnet (ebd., S. 128) und Goethes Verhältnis zum Orient als Exempel beschreibt ,für die von Said eingeräumte Möglichkeit, daß ein unabhängigerer oder skeptischerer Denker andere Ansichten der Sache $<$ des Orients $>$ haben konnte." "(ebd., S. 127f.).
} 
Untersucht man nun die konkreten im West-östlichen Divan nachweisbaren Übersetzungsstrategien, bleibt das Resultat zunächst widersprüchlich. Zuerst wäre festzuhalten, daß Übersetzungen für den West-östlichen Divan schon als Voraussetzung seiner Entstehung eine große Rolle spielen - wie Goethe in den Noten und Abhandlungen deutlich gemacht hat und wie wir ja auch aus der Entstehungsgeschichte des West-östlichen Divans wissen, die mit der Lektüre der gerade erschienenen ersten vollständigen Übersetzung der Gedichtsammlung (pers. dīwān) des großen persischen Dichters Hafis in einer Kutsche beginnt ${ }^{26}$. Unter den Gedichten des Goetheschen Divan selbst gibt es jedoch nur ganz wenige, die als Übersetzungen gelten können. Zwar lassen sich für zahlreiche Gedichte überwiegend orientalische Quellen nachweisen; um Übersetzungen in einem engerem Sinne handelt es sich jedoch nur selten. Die einzige umfangreiche Übersetzung der Sammlung ist das Gedicht Der Winter und Timur aus dem Buch Timur. Wie bei den freieren Bearbeitungen handelt es sich auch hier um eine Übertragung, aus zweiter Hand'. Das arabische Gedicht, das Goethes Der Winter und Timur zugrundeliegt, hat Goethe aus einer lateinischen Fassung weiterübersetz ${ }^{27}$, anderes übernimmt er aus dem Französischen seiner orientalistischen Quellen wie z.B. den Fundgruben des Orients $^{28}$, oder auch aus dem Deutschen der Hafis-Übertragung Joseph von Hammers. ${ }^{29}$ Dabei werden ganz verschiedene Verfahren des Übertragens eingesetzt - die Skala reicht von der seltenen wörtlichen Übernahme über die freie Bearbeitung bis zur produktiven Weiterverwendung einzelner Verse oder Motive.

So sind auch die von Witte herangezogenen Beispiele Selige Sehnsucht und Der Winter und Timur ausgehend von Goethes Typologie unterschiedlich einzuordnen: während es sich bei dem letzteren tatsächlich um eine weitgehend wörtliche Übersetzung aus dem Lateinischen handelt, läßt sich das erste nur in einem sehr weiten Sinn überhaupt als Übersetzung charakterisieren. So handelt es sich bei allen fünf Gedichten, die sich im Stadium des Wiesbadener Registers schon im Titel ausdrücklich auf Ghaselen von Hafis beziehen, durchaus nicht um Übersetzungen 'der dritten Art', bei denen sich der Übersetzer ,fest

\footnotetext{
${ }^{26}$ Joseph von Hammer: Der Diwan von Mohammed Schemsed-din Hafis. Aus dem Persischen zum erstenmal ganz übersetzt. Stuttgart / Tübingen 1812 [tatsächlich beide Bände erst 1814], Bde. 2.

${ }^{27}$ Zugrundeliegende Quelle ist die in arabischer Reimprosa abgefasste Timur-Biographie des Ibn 'Arabsah. Auszüge daraus kannte Goethe aus William Jones' Poeseos Asiaticae Commentariorum. Bei dem Gedicht Der Winter und Timur handelt es sich um eine fast wortgetreue Übertragung eines Abschnitts der lateinischen Prosavorlage in reimlose vierhebige Trochäen. Vgl. FA I, 3, S. 1163-1165 sowie Peter Michelsen, Goethes Gedicht „, Der Winter und Timur" und seine Vorlage. In: Formen innerliterarischer Rezeption. Hg. V. Wilfried Floeck, Dieter Steland, Horst Turk. Wiesbaden 1987, S. 445-458.

${ }^{28}$ Fundgruben des Orients, bearbeitet durch eine Gesellschaft von Liebhabern, 6 Bde., Wien 1809-1818.

${ }^{29}$ So z.B. die beiden berühmten Gedichte Erschaffen und Beleben und Selige Sehnsucht (Vgl. FA I, 3, S. 18 und 24f. mit Kommentar) und auch das Motto zum Buch Hafis, das Hammers Übersetzung der Schlußverse von Hafis' Ghasel Dal 109 in überarbeiteter Form aufnimmt.
} 
an sein Original anschließt" [FA I, 3.1, S. 281], sondern um freie Weiterdichtungen Hammerscher Hafis-Übertragungen, bei denen auch eine Einordnung als 'parodistische' Übersetzungen schwer fiele: häufig verbindet Goethes Gedicht mit dem Prätext nicht mehr als ein motivischer Anklang.

Trotzdem meine ich, daß Bernd Witte recht hat, wenn er konstatiert, daß Goethe mit den 'Übersetzungen der dritten Art' auch auf seinen eigenen West-östlichen Divan zielt. Freilich nicht das einzelne übersetzte Gedicht in seinem Verhältnis zum jeweiligen Original, sondern die Gedichtsammlung als Ganze trägt „die Zeichen ihrer Herkunft aus dem Fremden an sich“30. Das gilt nicht nur in Bezug auf ihre vielfältigen orientalischen Prätexte bzw. auf die östliche Kultur. Wie die das Fremde signalisierenden Elemente aus Hegire (die Wendung „Chisers Quell“ etwa oder der Ausdruck „Huris“, FA I, 3.1, S. 12f.) beziehen sich die meisten solcher Signale im West-östlichen Divan weniger auf ganz bestimmte Ghaselen bei Hafis, als auf fremdkulturellen Text im weiteren Sinn. Das gilt auch für den Einsatz fremdsprachlicher Elemente aus dem westlichen europäischen Kontext. Witte verweist überzeugend auf ungewöhnlich , anglisierte' Wortformen wie Schawl, Kriegesthunder, bewhelmen, (FA I, 3.1, S. 12, 19 und 86) und auf die französische Vokabel Hegire als Titel des prominenten Eröffnungsgedichts ${ }^{31}$, die hier ganz wie die Übersetzungen der 'dritten Art' als Zeichen dafür stehen, „daß Kultur niemals monolingual ist, sondern aus vielen Wurzeln erwächst ${ }^{\star 32}$ oder, so formuliert es der russische Kulturphilosoph Michail Bachtin, ,daß jede Einsprachigkeit im Grunde relativ ist. Die eigene einzige Sprache ist ja nicht allein: in ihr gibt es stets Überreste und Möglichkeiten der Anderssprachigkeit [... $]^{633}$.

Im Kontext seiner Überlegungen zur 'Weltliteratur' betont Goethe, daß ein wesentlicher Gewinn von Übersetzungen nicht die Bereicherung der Zielkultur

\footnotetext{
${ }^{30}$ Witte (Anm. 23) S. 89.

${ }^{31}$ Vgl. auch Wittes einleuchtende Beobachtungen zu Goethes Verfahren einer „Bedeutungserweiterung der eigenen Sprache durch Übersetzung“, die er am Beispiel der Herkunft des Goetheschen Neologismus ,stängeln“ aus dem Französischen erläutert. (Sommernacht, FA I $3,112)$.

${ }^{32}$ Witte (Anm. 23) S. 91.

${ }^{33}$ Michail Bachtin: Aus der Vorgeschichte des Romanwortes. In: Die Ästhetik des Wortes. Hrsg. von Rainer Grübel. Aus dem Russischen übersetzt von Rainer Grübel und Sabine Reese. Frankfurt/M. 1979, S. 301-337, hier S. 323. Ein Übersetzungsverfahren, das die nicht mehr wahrgenommene natürliche Hybridität von Sprache (und Kultur!), eben die „Überreste und Möglichkeiten der Anderssprachigkeit“" auf vielfältige Weise an die Oberfläche kehrt, verwandelt die Sprache - mit den Worten Bachtins - ,vom absoluten Dogma, das sie im Rahmen von Abgeschlossenheit und tauber Einsprachigkeit bildet, in eine Arbeitshypothese des Begreifens und Ausdrückens der Realität. [Ja,...] die wechselseitige Erhellung mit einer andern Sprache beleuchtet und objektiviert im Prozeß des literarischen Schaffens gerade die 'weltanschauliche' Seite der eigenen (und der fremden) Sprache, ihre innere Form, das ihr eigene System der Bewertung und Akzentuierung.“ (Ebd. S. 319) Und mit dieser ,aktiven wechselseitigen Erhellung der Sprachen und Kulturen“ tritt an die Stelle ,der einheitlichen und einzigen, abgeschlossenen, ptolemäischen sprachlichen Welt die offene galileische Welt der vielen, sich gegenseitig beleuchtenden Sprachen.“ (Ebd. S. 322)
} 
um ihr bis dahin unbekannte literarische Schätze sei, sondern daß ein wesentlicher Gewinn für die Ausgangskultur darin liegen kann, das Eigene in fremder Gestalt wiederzusehen. Die Erfahrung, daß die fremde Perspektive, die Spiegelung des Eigenen im Fremden in Gestalt von Übersetzungen oder auch von Besprechungen oder Gesprächen, zu veränderter Wahrnehmung und Klärung des Eigenen verhelfen kann, ist ein zentraler Gedanke der Goetheschen Überlegungen zur 'Weltliteratur'. ${ }^{34}$ Solchen 'Verfremdungseffekt' bestimmt er ganz ana$\log$ an anderer Stelle dann auch als den ,eigentlichste[n] Gewinn der Reisen“: „Das Bekannte wird neu durch unerwartete Bezüge, und erregt, mit neuen Gegenständen verknüpft, Aufmerksamkeit, Nachdenken und Urteil““. (FA I, 24, S. 747)

Diese ,ins Ganze greifende Bemerkung“ (ebd., S.746) stammt bemerkenswerterweise nicht aus einem der bekannten Reiseberichte Goethes, sondern aus der Geschichte seiner botanischen Studien - und es lohnt sich ein Blick auf diesen Kontext. Es geht hier um morphologische Studien, genauer um die Metamorphose der Pflanzen, und Goethe schildert seine Reiseerfahrungen in diesem Zusammenhang als Voraussetzung und Auslöser für seine Entdeckung der 'Urpflanze'. ${ }^{35}$ Er berichtet von seiner Beobachtung,

daß alles was uns von Jugend auf umgab, jedoch nur oberflächlich bekannt war und blieb, stets etwas Gemeines und Triviales für uns behält, das wir als gleichgültig neben uns bestehend ansehen, worüber zu den-

\footnotetext{
${ }^{34}$ Daß für Goethe dieser Effekt von Übersetzungen im Mittelpunkt seiner 'Weltliteratur'-Idee steht, wird sehr plastisch z.B. in einem weiteren Schreiben an Thomas Carlyle, dem er über eine Übersetzung des Schillerschen Wallenstein - eine Übersetzung nicht von Carlyle selbst sondern von dessen Landsmann George Moir - im Juni 1828 folgendes schrieb: „Die Übersetzung des Wallensteins hat auf mich einen ganz eignen Eindruck gemacht, da ich die ganze Zeit, als Schiller daran arbeitete, ihm nicht von der Seite kam, zuletzt, mit dem Stück völlig bekannt, solches vereint mit ihm auf das Theater brachte, allen Proben beywohnte und dadurch mehr Qual und Pein erlebte als billig, die nachfolgenden Vorstellungen nicht versäumen durfte, um die schwierige Darstellung immer höher zu steigern; so läßt sich's denken, daß dieses herrliche Stück mir zuletzt trivial, ja widerlich werden mußte; auch hab ich es in zwanzig Jahren nicht gesehen und nicht gelesen. Nun aber da ich es unerwartet in Shakespeare's Sprache wieder gewahr werde, so tritt es auf einmal wie ein frisch gefirnißtes Bild in allen seinen Theilen wieder vor mich, und ich ergötze mich daran wie vor Alters und noch dazu auf eine ganz eigene Weise. Sagen Sie das dem Übersetzer [...] Hier aber tritt eine neue, vielleicht kaum empfundene, vielleicht nie ausgesprochene Bemerkung hervor: daß der Übersetzer nicht nur für seine Nation allein arbeitet, sondern auch für die aus deren Sprache er das Werk herüber genommen. Denn der Fall kommt öfter vor als man denkt, daß eine Nation Saft und Kraft aus einem Werke aussaugt und in ihr eigenes inneres Leben dergestalt aufnimmt, daß sie daran keine weitere Freude haben, sich daraus keine Nahrung weiter zueignen kann. Vorzüglich begegnet dieß den Deutschen, die gar zu schnell alles was ihnen geboten wird verarbeiten und, indem sie es durch mancherlei Wiederholungen umgestalten, es gewissermaßen vernichten. Deshalb denn sehr heilsam ist, wenn ihnen das Eigne durch eine wohlgerathene Übersetzung späterhin wieder als frisch belebt erscheint. (Goethe an Carlyle, 15. 6. 1828 [ähnlich in einer Besprechung, die Goethe in Kunst und Alterthum veröffentlichte]. WA IV, 44, S. 139f.)

35 „Der Verfasser teilt die Geschichte seiner botanischen Studien mit“. Nachtrag zum Versuch die Metamorphose der Pflanzen zu erklären. In: Deutsch-französische Ausgabe des Versuchs über die Metamorphose der Pflanzen (FA I, 24, S. 732-752).
} 
ken wir gewissermaßen unfähig werden. Dagegen finden wir, daß neue Gegenstände in auffallender Mannigfaltigkeit, indem sie den Geist erregen, uns erfahren lassen daß wir eines reinen Enthusiasmus fähig sind; sie deuten auf ein Höheres, welches zu erlangen uns wohl gegönnt sein dürfte. (FA I, 24, S. 746)

Es folgt die oben schon zitierte Passage: „Dies ist der eigentlichste Gewinn der Reisen, und jeder hat nach seiner Art und Weise genugsamen Vorteil davon. Das Bekannte wird neu durch unerwartete Bezüge, und erregt, mit neuen Gegenständen verknüpft, Aufmerksamkeit, Nachdenken und Urteil““ (ebd.). Und er fährt fort:

In diesem Sinne ward meine Richtung gegen die Natur, besonders gegen die Pflanzenwelt, bei einem schnellen Übergang über die Alpen lebhaft angeregt [...]. Ich ging allen Gestalten, wie sie mir vorkamen, in ihren Veränderungen nach, und so leuchtete mir am letzten Ziel meiner Reise, in Sicilien, die ursprüngliche Identität aller Pflanzenteile vollkommen ein, und ich suchte diese nunmehr überall $\mathrm{zu}$ verfolgen und wieder gewahr zu werden. (FA I, 24, S. 747)

Von dieser im botanischen Garten von Palermo erfahrenen „Erleuchtung über botanische Gegenstände“" (FA I 15.1, S. 239) hat Goethe auch in der Italienischen Reise berichtet: „Im Angesicht so vielerlei neuen und erneuten Gebildes fiel mir die alte Grille wieder ein: ob ich nicht unter dieser Schar die Urpflanze entdecken könnte?" (FA I 15.1, S. 286) ${ }^{36}$

Wie der Blick auf die fremden Pflanzen als Katalysator wirkte für die Erkenntnis der „sinnlichen Form einer übersinnlichen Urpflanze“ (FA I 24, S. 748) als einem aller Pflanzenbildung zugrundeliegenden Gesetz, so zielt der West-östliche Divan als 'poetische Orientreise' - und als 'Weltliteratur' avant la lettre - auf das entsprechende poetische 'Urphänomen'. Den Begriff 'Urpoesie' hat Goethe dabei nirgends gebraucht, in direktem Kontext der ersten öffentlichen Verwendung des 'Weltliteratur'-Begriffs ist dagegen von „Weltpoesie“ die Rede. In einer Besprechung neuer deutscher Übersetzungen serbischer Volkspoesien, die von Goethe mit Gedichten des zeitgenössischen französischen Dichters Pierre Jean de Béranger verglichen werden, heißt es:

Auffallend mußte hiebey seyn daß ein halbrohes Volk mit dem durchgeübtesten gerade auf der Stufe der leichtfertigsten Lyrik zusammentrifft, wodurch wir uns abermals überzeugen daß es eine allgemeine Weltpoesie gebe und sich nach Umständen hervorthue; weder Gehalt noch Form braucht überliefert zu werden, überall wo die Sonne hin-

${ }^{36}$ „Eine solche muß es denn doch geben! Woran würde ich sonst erkennen, daß dieses oder jenes Gebilde eine Pflanze sei, wenn sie nicht alle nach einem Muster gebildet wären." Italienische Reise, Palermo, 17.4.1787 (WA I 15, S. 286). 
scheint ist ihre Entwicklung gewiß (Ueber Kunst und Alterthum VI 1. FA I, 22, S. 386).

Im Gegensatz zu dem Phänomen aktueller internationaler Kommunikation, das Goethe als 'Weltliteratur' bezeichnet, bezeichnet 'Weltpoesie' - in deutlicher Analogie zur 'Urpflanze' - die „Eine Dichtung, die ächte“ (Ueber Kunst und Alterthum $V$ 3. FA I, 22, S. 287), die ,ein Gemeingut der Menschheit ist“ und „überall und zu allen Zeiten in Hunderten und aber Hunderten von Menschen hervortritt"“ (zu Eckermann, 31. 1. 1827. FA II, 12, S. 224). ${ }^{37}$

Die Überzeugung, daß „Poesie eine allgemeine Welt- und Völkergabe sei“, die Goethe nach seinem Bericht in Dichtung und Wahrheit in Straßburg von Herder übernommen hatte ${ }^{38}$, bildet die Basis seiner Idee einer 'Weltliteratur' und die Voraussetzung für Goethes hermeneutische Konzeption des 'Fremdverstehens', wie sie dem West-östlichen Divan und der 'Übersetzung der dritten Art' zugrundeliegt. ${ }^{39}$ Eine der 'Urpflanze' vergleichbare sinnliche Konkretisierung der allgemeinen Idee in einem symbolischen Phänomen hat Goethe im Fall der Poesie freilich nicht gefunden zu haben geglaubt. Als paradigmatische Zeugen für das universale Phänomen 'Weltpoesie' aber verweist er in der einschlägigen Passage aus Dichtung und Wahrheit auf ganz konkrete Beispiele: neben der „Volkspoesie, deren Überlieferungen im Elsaß aufzusuchen er [Herder] uns antrieb“ nennt er die „hebräische Dichtkunst“. (FA I, 14, 454) Auf die Bibel als „der ältesten Sammlung“ orientalischer Poesie kommt Goethe keineswegs zufällig auch ganz zu Beginn der Noten und Abhandlungen des West-östlichen Divans zu sprechen und gedenkt hier ausdrücklich eben ,jener Zeit wo Herder und Eichhorn uns hierüber persönlich aufklärten [als] [...] eines hohen Genusses, dem reinen orientalischen Sonnenaufgang zu vergleichen." (West-östlicher Divan. FA I, 3, S. 140). Zwei Bücher des Alten Testaments greift Goethe im folgenden heraus: neben dem Buch Ruth als Inbegriff einer epischen Idylle kommt er hier auf das ,hohe Lied“" zu sprechen ,,als dem zartesten und unnachahmlichsten was uns von Ausdruck leidenschaftlicher, anmuthiger Liebe zugekommen“ (Ebd.). Eben dieses Beispiel hebräischer Dichtkunst hatte Goethe

\footnotetext{
${ }^{37}$ Der von Goethe nicht eindeutig bestimmte Begriff, Weltliteratur' meint bei ihm vorwiegend den Vorgang - des Reisens, der Übersetzung -, nicht das Resultat solcher Tätigkeiten. Ziel wäre also nicht Weltliteratur (etwa im Sinne eines Kanons), sondern die Erkenntnis von $>$ Urpoesie $<-$ nicht als Selbstzweck, sondern als Ermöglichungsgrund des Verstehens des Fremden.

38 „Die hebräische Dichtkunst, welche er [Herder] nach seinem Vorgänger Lowth geistreich behandelte, die Volkspoesie, deren Überlieferungen im Elsaß aufzusuchen er uns antrieb, die ältesten Urkunden als Poesie, gaben das Zeugnis, daß die Dichtkunst überhaupt eine Weltund Völkergabe sei, nicht ein Privaterbteil einiger feinen, gebildeten Männer" (Dichtung und Wahrheit, FA I, 14, S. 445).

${ }^{39}$ Vgl. Manfred Koch: Weimaraner Weltbewohner. Zur Genese von Goethes Begriff „,Weltliteratur“. Tübingen 2002, S. 266 (Fußnote 85): „Das ,allgemein Menschliche’ ist auch für Goethe weniger die substantielle Gleichheit der Lebensformen als ihre wechselseitig Verstehbarkeit in der Differenz (die allerdings basiert auf einem Kernbestand an Universalien [...]“).
} 
wenige Jahre nach der Straßburger Begegnung mit Herder selbst übersetzt. Die als Handschrift erhaltene Übertragung zeigt Goethes Bemühen um eine philologisch treue Übersetzung und gleichzeitig das Bestreben, den Text sich anzueignen, ein deutsches Gedicht eigenen Rechts daraus zu gewinnen. ${ }^{40}$ Viel später nennt er diese biblische Poesie dann in einem der 'Weltpoesie' gewidmeten Gedicht aus dem 'Weltliteraturheft' seiner Zeitschrift Über Kunst und Alterthum. Hier steht „der Winzerin Lied“ in einer Reihe mit den Psalmen Davids, den Ghaselen des Hafis und den Liedern südamerikanischer Ureinwohner. $^{41}$

Auf dieses Beispiel „naiver Dichtkunst“ (FA I, 3, S. 140) kommt Goethe nicht nur im Prosateil des West-östlichen Divans zurück. Das Hohe Lied hat auch in den Gedichten des West-östlichen Divans seine Spuren hinterlassen. Auf ganz andere Art als die philologisch-wörtliche Übersetzung des jungen Goethe läßt sich auch das Buch Suleika als produktive Aufnahme und Spiegelung auffassen dieser ,herrlichste[n] Sammlung Liebeslieder, die Gott erschaffen hat“ (an Merck am 11.10.1775. WA IV, 2, S. 299). Wörtliche Anklänge an den Text des Hohen Liedes lassen sich hier kaum finden, allenfalls im Gedicht Vollmondnacht könnte man solche vermuten. Der Refrain „Ich will küssen! Küssen sagt' ich“ und die Formulierung „Lieblicher als Weines Lippen“ erinnern durchaus an den Eingang des seit Luther im Deutschen als Hohes Lied vertrauten Liedes der Lieder: „Er küsse mich | Mit seines Mundes Küssen | Denn deine Liebe ist lieblicher als Wein“ lauten die beiden ersten Verse in der 1778 erschienenen Übertragung Herders. ${ }^{42}$ Das Verhältnis des Buches Suleika zum Hohen Lied Salomos ist jedoch nicht das einer Übersetzung oder Weiterdichtung, es handelt sich aber auch nicht lediglich um die Aufnahme einzelner Motive, sondern es geht um die Aufnahme und produktive Anwendung eines bestimmten Modells von Liebesdichtung, wie es im Hohen Lied paradigmatisch realisiert ist. Ganz deutlich nämlich sind eine Reihe von strukturellen Analogien: Wie das Lied der Lieder bietet das Buch Suleika eine Sammlung von Einzelgedichten, in denen sich ein vom Sich-Finden, Sich-Verlieren und Wiederfinden der Liebenden geprägtes „Duodrama“ zwischen den Protagonisten abspielt ${ }^{43}$, das in

\footnotetext{
${ }^{40}$ Vgl. FA I, 12, S. 364-370 und Kommentar.

41 „Wie David königlich zur Harfe sang, | Der Winzerin Lied am Throne lieblich klang,| Des Persers Bulbul Rosenbusch umbangt, | Und Schlangenhaut als Wildengürtel prangt, | Von Pol zu Pol Gesänge sich erneuen - | Ein Sphärentanz harmonisch im Getümmel - | Lasst alle Völker unter gleichem Himmel | Sich gleicher Gabe wohlgemuth erfreun!“ (FA I, 22, S. 390)

${ }^{42}$ Johann Gottfried Herder, Lieder der Liebe. Die ältesten und schönsten aus Morgenlande. In: ders., Volkslieder, Übertragungen, Dichtungen. Hg. Ulrich Gaier (Bd. 3 der Werk-Ausgabe des Deutschen Klassiker Verlags), Frankfurt/Main 1990, S. 431-521, hier S. 434. In der Übersetzung Goethes lauten die Verse: „Küß er mich den Kuß seines Mundes! Trefflicher ist deine Liebe denn Wein“" (FA I, 12, S. 364).

43 Das Lied der Lieder hat im Laufe seiner reichen Rezeptionsgeschichte immer wieder zur Rekonstruktion in dramatischer Form herausgefordert; auch die von Goethe 1820 wohlwol-
} 
beiden Fällen die Rezipienten wiederholt zum Versuch einer Rekonstruktionen des ,ursprünglichen' Handlungszusammenhangs motiviert hat. Die entscheidende Gemeinsamkeit aber ist die grundlegend dialogische Struktur beider Gedichtsammlungen. Es verbindet sie die in der Geschichte der Liebesdichtung keineswegs häufig anzutreffende Tatsache, daß beide Stimmen zu hören sind; im Hohen Lied wie im Buch Suleika sind die Liebenden einander ebenbürtige Partner, die als gleichberechtigte Sprecher einen Dialog führen. ${ }^{44} \mathrm{Da}$ in beiden Dichtungen als dritte Sprecherinstanz zusätzlich eine Gruppe junger Frauen auftritt - die „Töchter Jerusalems“ im Hohen Lied und die „Mädchen“ im Buch Suleika - verstärkt die strukturelle Analogie. ${ }^{45}$

Darüber hinaus aber sind die Lieder Salomos und diejenigen des Westöstlichen Divans vor allem auch durch die Vermittlung einer Liebeserfahrung verbunden, die von konkreter zwischenmenschlicher und sinnlich-körperlicher Liebe ins Geistige, Symbolisch-transzendente weist: „der Schleier irdischer Liebe scheint höhere Verhältnisse zu verhüllen“"46. Diese Charakterisierung des Buch Suleika aus Goethes Ankündigung des West-östlichen Divans im Morgenblatt für gebildete Stände läßt sich ohne weiteres auch für das Hohe Lied in Anspruch nehmen, das in der abendländischen Tradition wohl als Inbegriff aller zwischen sinnlich-erotischer und geistig-symbolischer Liebesauffassung vermittelnder Dichtung gelten kann.

Haben wir uns von unserem Thema jetzt ganz entfernt? Was hat das alles noch mit dem West-östlichen Divan als Reise, mit dem West-östlichen Divan als 'Weltliteratur' zu tun?

Nun, die vorgestellte produktive Wiederaufnahme des Hohen Liedes im Buch Suleika scheint mir ein Beispiel für das den West-östlichen Divan wie das Konzept 'Weltliteratur' prägende Verfahren der 'wiederholten Spiegelungen' zu bieten, ein Verfahren, das in Analogie zu Goethes Erfahrungen mit der 'Urpflanze' das Allgemeine und Gesetzmäßige gerade in der Wahrnehmung der Vielfalt und im Austausch mit dem Fremden erkennbar werden läßt. Dieses

lend besprochene Bearbeitung Umbreits gehört zu den zahlreichen Bearbeitungen, die aus der Liedersammlung ein dramatisches Geschehen gestalten. Für das Buch Suleika unternahm H. A. Korff den Versuch, die „Liebesgedichte des West-östlichen Divans in zeitlicher Folge“ zu edieren und so den ,Zusammenhang des poetischen Romans“, den Goethes Anordnung absichtlich verschleiere, wieder sichtbar zu machen (Leipzig, Stuttgart, Zürich 1947).

${ }^{44} \mathrm{Da}$ die Geliebte ,,persönlich als Dichterinn auftritt“" nennt Goethe selbst als charakteristisches Merkmal des Buch Suleika (in der Ankündigung des Divans im Morgenblatt für gebildete Stände, FA I, 3, S. 550), das den Dialog zwischen Suleika und Hatem enthält. Daß einige der schönsten hier versammelten Gedichte tatsächlich Gedichte Marianne von Willemers sind, blieb lange unbekannt. Der Sprecherwechsel ist charakteristisches Merkmal auch des Hohen Liedes, Lieder der weiblichen Liebenden wechseln sich hier ab mit solchen aus männlicher Perspektive.

${ }^{45}$ Die „Töchter Jerusalems“ werden im Hohen Lied wiederholt angesprochen; sie sprechen selber reagieren in V. 9-16 VI,1-2. Im Buch Suleika vgl. das Zwiegespräch zwischen „Hatem“ und den ,Mädchen“ (FA I,3, S. 85-87).

${ }^{46}$ Morgenblatt für gebildete Stände. In: West-östlicher Divan (FA I, 3, S. 550). 
Verfahren ließe sich für den West-östlichen Divan selbstverständlich in erster Linie auch im Umgang mit der persischen Dichtung zeigen. Erkennbar wird 'das Allgemeine' allerdings hier nicht in dem Sinne, daß es sich festhalten und bestimmen ließe, eine Definition oder Demonstration von $>$ Urpoesie $<$ also möglich wäre. Denn für solche müßte wohl gelten, was Goethe nach dem Bericht Eckermanns über „das Schöne“ gesagt hat: Es ist „, ...> ein Urphänomen, das zwar nie selber zur Erscheinung kommt, dessen Abglanz aber in tausend verschiedenen Äußerungen des schaffenden Geistes sichtbar wird und so mannigfaltig und so verschiedenartig ist als die Natur selber." (Goethe zu Eckermann, 18.4.1827, FA II, 12, S. 598)

Goethes Überlegungen zur Weltliteratur lassen erkennen, daß es ihm nicht darum geht, die Besonderheiten der jeweils unterschiedlichen Sprachen, Länder und Kulturen im Interesse eines 'Allgemein-Menschlichen' zu überwinden. Gerade umgekehrt bildet die Annahme eines solchen Allgemeinen aber die Voraussetzung für die Möglichkeit jedes Fremdverstehens - ohne daß der mit dem Austausch in Gang gesetzte Spiegelungsprozeß, der in ,jedem Besonderen [...] durch Nationalität und Persönlichkeit hin jenes Allgemeine immer mehr durchleuchten und durchscheinen “47 lasse, zum Stillstand eines endgültigen Ergebnisses gebracht werden könnte. 'Weltliteratur' bezeichnet im Sinne Goethes gerade nicht das zukünftige Resultat eines Vorgangs, das als ein Allgemeines an die Stelle der Besonderheiten träte, sondern den das Besondere im Hinblick auf das 'allgemein Menschliche' reflektierenden Spiegelungsprozeß selbst. Das postulierte 'Allgemein-Menschliche' übernimmt für diesen Vorgang die Funktion einer 'regulativen Idee', die sich aus den miteinander in Kontakt kommenden verschiedenen besonderen Standpunkten immer wieder neu verhandeln lassen muß.

Mit solchen Überlegungen scheint mir Goethe 'in interkultureller Perspektive' durchaus bis heute aktuell.

${ }^{47}$ German Romance. In: Kunst und Alterthum VI 2 (FA I, 22, S. 433). 